\title{
Reduced Complexity Tree Search Algorithms for MIMO Decoding
}

\author{
Gajanan R Patil, and Vishwanath K Kokate
}

\begin{abstract}
Maximum Likelihood Decoding (MLD) is computationally complex technique for decoding received information in multiple input multiple output (MIMO) systems. Tree search algorithms such as sphere decoding (SD) and QR decomposition with $M$ survivals (QRD-M) are used to reduce the complexity keeping the performance near ML. This paper presents two techniques for reducing the computational complexities of the tree search algorithms further. The first technique is based on selecting the initial radius for sphere decoding. The main contribution of this paper is that the greedy best first search is used to compute initial radius, instead of Babai estimate. The second contribution is, QRD-M algorithm is modified to prune the nodes in the current layer based on maximum metric of child nodes of smallest surviving node. The performance of the proposed techniques is tested for different MIMO systems in terms of bit error rates (BER) and average number of nodes visited. The proposed schemes have improved computational complexity with no degradation of performance.
\end{abstract}

Index Terms-Best First Search, Maximum Likelihood Decoding, MIMO, QRD-M, Sphere decoding.

\section{INTRODUCTION}

The need for reliable, high speed wireless communication is fulfilled by MIMO technology. MIMO based communication system consists of multiple antennas at both transmit and receive ends. Several techniques have been proposed that utilize the MIMO channel in different ways. The MIMO schemes can be classified as spatial multiplexing, spatial diversity (space-time coding) and smart antennas \& beamforming techniques [1] [2].

In spatial multiplexing (SM) based MIMO system, the data to be transmitted is demultiplexed and transmitted on different antennas. At the receiver end, a detection technique, capable of separating the transmitted signals with acceptable complexity and better performance is required. Several MIMO detection techniques are proposed in the literature. ML detection technique is found to have an optimum performance but the complexity of the algorithm increases exponentially with number of transmit antennas and order of modulation which makes it infeasible for practical implementation [3]. To reduce the computational complexity, tree search algorithms are proposed which can achieve quasi-ML performance. Sphere decoding and QRD-M algorithms are the two main tree search algorithms.

Manuscript received October 04, 2014; revised December 13, 2014

G. R Patil is with the Dept of Electronics \& Telecommunication Engg. Army institute of Technology Pune, India and with the Dept of Electronics \& Telecommunication Engg. Sinhgad College of Engg, Pune, India (e-mail: patilgr67@yahoo.co.in).

V. K Kokate is with the Dept of Electronics \& Telecommunication Engg. Sinhgad College of Engg. Pune, India (e-mail: vkkokateetc@gmail.com).
Sphere decoding was originally presented in [4] by Fincke and Pohst called FP strategy. The algorithm was modified by Schnorr and Euchnerr in [5] called SE strategy. Sphere decoding is based on searching for a point in the lattice from the received signal point. The search is limited within a sphere of radius 'd' instead of searching in the entire lattice space [6]. The choice of this radius is very crucial in deciding the performance of the algorithm. A too large value of ' $d$ ' will increase the complexity, whereas a too small value will fail to capture a point inside the sphere. One of the method suggested in [7] for choice of ' $d$ ' is the Babai estimate which guarantees existence of at least one point inside the sphere. The Babai estimate is obtained using least square (LS) detection. This method also enhances the noise, which in turn increases the initial search radius. The increase in initial search radius leads to increase in computational complexity. In this paper, we have proposed a technique based on best first search, which avoids enhancement of noise due to LS estimate, at the same time, guarantees existence of at least one point inside the sphere. The simulation results in section IV demonstrate that the choice of initial radius using best first search, leads to less computational complexity than the choice of radius using Babai estimate. Simplified implementation of sphere decoder using depth first search (DFS) is discussed by the authors of this paper in [8] and the same algorithm is used to verify this.

The QRD-M algorithm gives quasi-ML performance and has fixed computational complexity. The QRD-M algorithm was originally discussed in [9]. It can reduce the tree search complexity by selecting only best $\mathrm{M}$ candidates at each layer instead of testing all the hypotheses of the transmitted symbol. These $\mathrm{M}$ candidates are selected such that they have the smallest accumulated metric values. A simplified implementation of QRD-M algorithm and a QRD-M with reduced $M$ algorithm using breadth first search (BFS) is discussed by the authors of this paper in [10]. Number of techniques for reduction in computational complexity of QRD-M algorithm is suggested in literature [11]-[20]. Fixedcomplexity sphere decoder (FSD) was proposed in [21] and [22] by Barbero et al. to overcome the drawbacks of sphere decoder. Fixed complexity k-best sphere decoders are discussed in [23] and [24]. Some techniques are proposed in literature for preprocessing of channel matrix before the detection is carried out to improve the detection performance [25]. A storage based approach which combines both depthfirst search and breadth-first search can be found in [26].

In this paper a modification of QRD-M algorithm is also proposed. In QRD-M algorithm the surviving nodes at each level/layer are fixed. In the proposed algorithm, these nodes are selected for expansion to the next level, only if its metric is less than the current maximum metric at hand. The current metric is selected based on the maximum metric of child nodes 
of smallest surviving node at the current layer. The simulation results in section IV indicate that the proposed method leads to improvement in computational complexity with no change in performance.

The rest of this paper is organized as follows. In section II, MIMO system model is described. The proposed algorithms are discussed in Section III. The simulated performance of MIMO system using these algorithms is presented in section IV. Finally, conclusion is given in section V.

\section{SYSTEM MODEL}

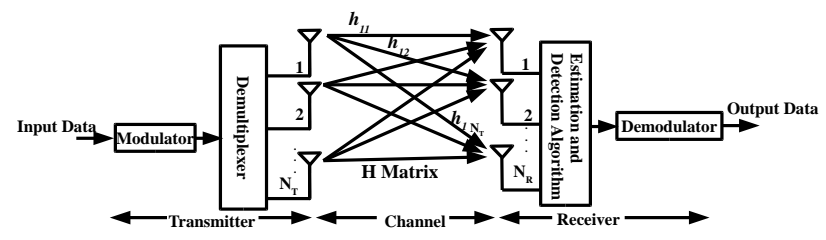

Fig.1. MIMO System with Spatial Multiplexing

Consider a Spatial Multiplexing based MIMO system shown in fig. 1 with $N_{T}$ transmit and $N_{R}$ receive antennas where, $N_{R} \geq N_{T}$. Let us assume the channel to be Rayleigh flat fading with channel matrix- $\boldsymbol{H} \in \mathbb{C}^{N_{R} \times N_{T}}$. Each element $h_{i j}$ in the matrix, represents the flat-fading channel coefficient between the $\mathrm{i}$-th receive and $\mathrm{j}$-th transmit antenna. Independent data streams are modulated and transmitted on $N_{T}$ different antennas. Denoting the complex received data by $\boldsymbol{r} \in$ $\mathbb{C}^{N_{R} \times 1}$ the equivalent base-band system can be modeled as,

$$
\boldsymbol{r}=\boldsymbol{H} \boldsymbol{x}+\boldsymbol{n}
$$

where, $\boldsymbol{x} \in \mathbb{C}^{N_{T} \times 1}$ is transmitted symbol vector and $\boldsymbol{n}$ is the additive white Gaussian noise with zero mean and noise power $\sigma_{n}^{2}$. Also, the sources are assumed to be spatially and temporally independent with identical source power $\sigma_{s}^{2}$.

\section{A. Sphere Decoding Algorithm}

The MLD estimate of transmitted symbol stream can be obtained as,

$$
\tilde{\mathbf{x}}=\arg \min \left\|\boldsymbol{r}-\boldsymbol{H} \boldsymbol{x}_{\boldsymbol{k}}\right\|^{2}
$$

Note that the channel matrix $\boldsymbol{H}$ is assumed to be known which otherwise has to be estimated. MLD compares received signal with all possible transmitted symbol streams which have undergone the Rayleigh fading.

The SD algorithm can solve the ML detection problem in (2) by searching over a restricted subset $\Omega$ that at least contains the ML solution. The channel matrix $\boldsymbol{H}$ is decomposed into $\boldsymbol{H}=\boldsymbol{Q R}$, where $\boldsymbol{R}$ is upper triangular and $\boldsymbol{Q}$ has orthogonal columns with unit norms. Hence system model in Eq. (1) can be written as,

$$
\boldsymbol{y}=\boldsymbol{R} \boldsymbol{x}+\boldsymbol{v}
$$

where, $\boldsymbol{y}=\boldsymbol{Q}^{H} \boldsymbol{r}$ and $\boldsymbol{v}=\boldsymbol{Q}^{H} \boldsymbol{n}$ and the ML problem in (2) can be written as,

$$
\tilde{\mathbf{x}}=\underset{\boldsymbol{x}_{\boldsymbol{k}} \in \Omega}{\arg \min }\left\|\boldsymbol{y}-\boldsymbol{R} \boldsymbol{x}_{\boldsymbol{k}}\right\|^{2}
$$

The sphere decoder solves (4) satisfying the constraint,

$$
\left\|\boldsymbol{y}-\boldsymbol{R} \boldsymbol{x}_{\boldsymbol{k}}\right\|^{2} \leq d^{2}
$$

Due to the upper triangular form of $\boldsymbol{R}$, the values of $\boldsymbol{x}$ can be solved form (4) level by level using substitution algorithm. Let $\mathrm{x} \mathrm{i}^{\wedge}\left(\mathrm{N} \_\mathrm{T}\right)=\left(\mathrm{x} \_\mathrm{i}, \mathrm{x} \_(\mathrm{i}+1), \ldots \mathrm{x} \_\left(\mathrm{N} \_\mathrm{T}\right)\right)$ denote last N_T-i-1 components of vector $\boldsymbol{x}$. The sphere search can be illustrated with a tree structure, where the algorithm aims at finding the shortest path between the root layer and the leaf layer. First, the last elements of the possible symbol vectors are calculated, i.e., $x_{N_{T}}$ and then $x_{N_{T}-1}$ and soon. The metric, squared Partial Euclidean Distance (PED) of $\boldsymbol{x}_{i}^{N_{T}}$ can be calculated as,

$$
d\left(\boldsymbol{x}_{i}^{N_{T}}\right)=d\left(\boldsymbol{x}_{i+1}^{N_{T}}\right)+\left|y_{i}-\sum_{j=i}^{N_{T}} r_{i j} x_{j}\right|^{2}
$$

Where the initial value of the metric, $d\left(x_{N_{T}}^{N_{T}}\right)=0$. Depending on the search strategy and the channel realization, the SD searches a variable number of nodes in the tree structure, and aims to find the point $\boldsymbol{x}=\boldsymbol{x}_{1}^{N_{T}}$, also called a leaf node, for which the Euclidean distance (ED) $d\left(\boldsymbol{x}_{1}^{N_{T}}\right)$ is minimum.

The SD algorithm for solving (4) can be implemented through a Depth First Search (DFS) tree traversal using (5) as pruning criteria to reduce the search. The node at $i$-th level represents a sequence of symbols from $x_{N_{T}-i+1}$ to $x_{N_{T}}$ counting from root of the tree which is at $N_{T}$-th level. All the leaf nodes in the tree will represent the full sequence $x_{1}$ to $x_{N_{T}}$. The algorithm searches all nodes in the tree that will satisfy constraint (5). As pointed out earlier, the initial choice of radius has direct impact on the performance and complexity of sphere decoding algorithm.

The adaptive sphere decoding algorithm instead of going through every point in the hyper sphere remembers the currently closest point and compares it with a new point to determine whether the new point is closer than the known one. This algorithm keeps on updating the sphere radius to avoid searching the unnecessary lattice points. The sphere decoding algorithm with radius update is given as Algorithm.1.

\section{B. QRD-M Algorithm}

QRD-M algorithm can reduce the tree search complexity by selecting only $\mathrm{M}$ candidates at each layer instead of testing all the hypotheses of the transmitted symbol. These $M$ candidates are the smallest accumulated metric values. The QRD-M algorithm can be implemented using Breadth first Search (BFS) technique and is given in Algorithm. 2.

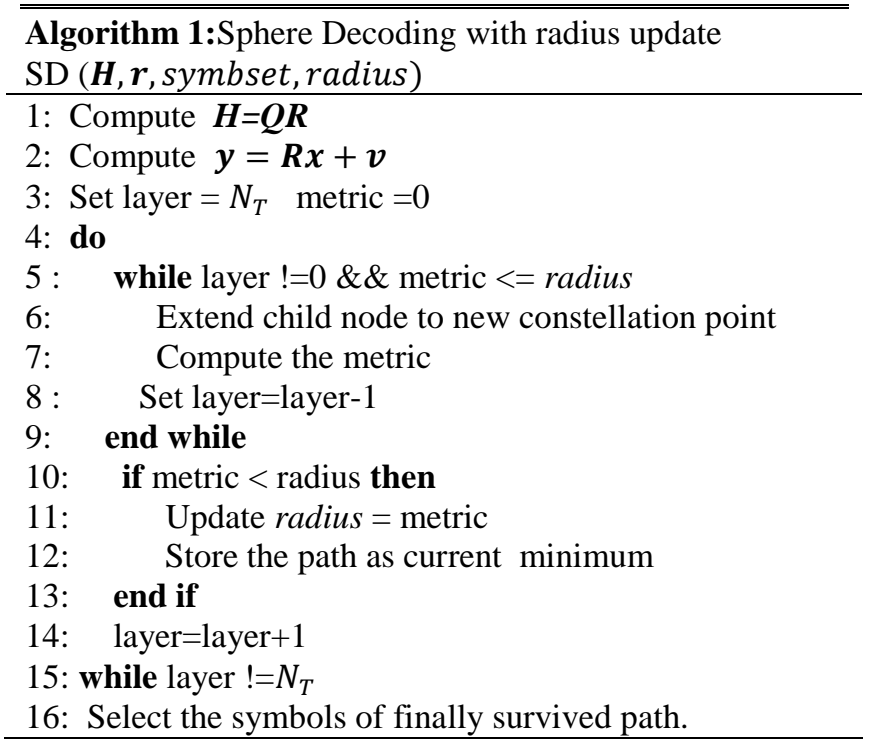




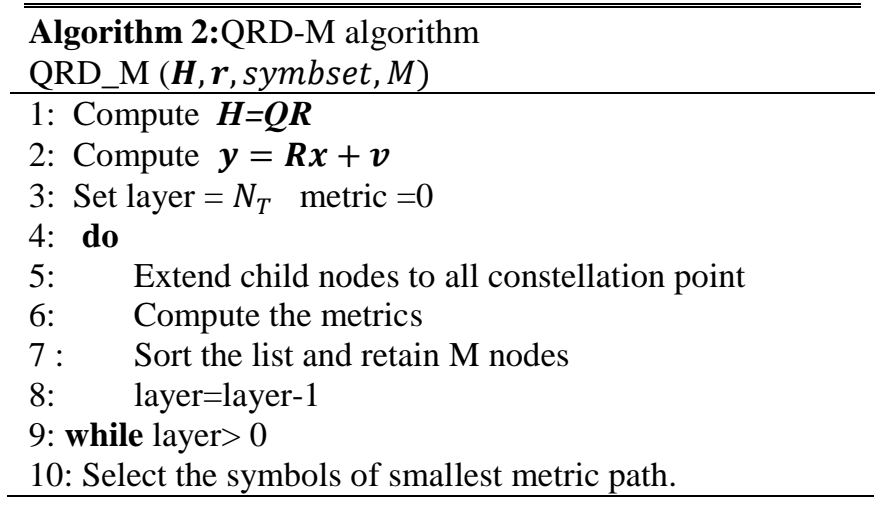

\section{C. $Q R D-M$ with Successive Reduction of $M$}

In [10] we proposed a novel scheme of QRD-M with successive reduction of $\mathrm{M}$ which requires reordering only once at the given layer similar to QRD-M, at the same time, number of survivals keeps on reducing at each layer. The FSD scheme can be modified to make $M$ variable at every level. For example we can start with $\mathrm{M}=4$ at level $N_{T}$, and then keep on dividing $\mathrm{M}$ by 2 in the subsequent levels. Thus computational complexity of FSD with progressive reduction of $\mathrm{M}$ is better than the simple QRD-M and FSD scheme. The modified algorithm is given as Algorithm. 3 .

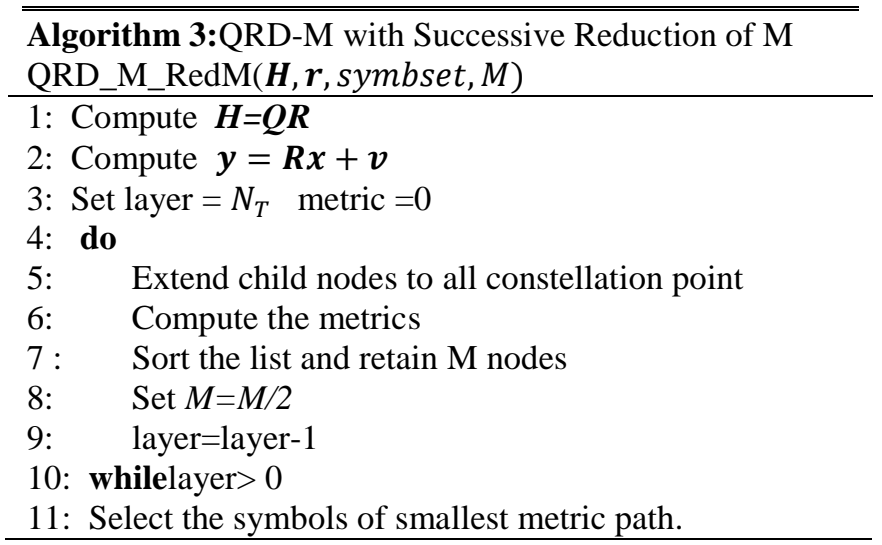

\section{PROPOSED ALGORITHMS}

In order to reduce the computational complexity of the algorithms discussed in section II, some modifications are suggested.

\section{A. Best First Search}

The sphere decoding algorithm can be speeded up if the initial choice of the radius ' $\mathrm{d}$ ' is correct. According to [7], the choice of ' $d$ ' can be distance between Babai estimate and vector $\boldsymbol{y}$ as,

$$
d=\left\|\boldsymbol{y}-\boldsymbol{H} \widehat{\boldsymbol{x}_{\boldsymbol{b}}}\right\|
$$

where, $\widehat{x_{\boldsymbol{b}}}$ is obtained using least square (LS) detection or zero forcing $(\mathrm{ZF})$ detection. The $\mathrm{ZF}$ detector also enhances the noise. Hence the initial estimate of the radius ' $\mathrm{d}$ ' is dependent mainly on noise. Thus drawback of Babai estimate is that it may give rise to loose initial point [26]. This can happen especially for low signal-to-noise ratio (SNR) values. Thus though this method guarantees at least one lattice point inside the sphere, there can be large number of points as well, leading to increased number of searches. In this work we are proposing an alternative method based on best first search.
The best first search method requires minimum number of computations to arrive at the choice of initial radius at the same time it eliminates drawback of Babai estimator. A lattice point nearer to received vector is obtained by searching through the tree layer by layer. The best child node with minimum metric is selected every time we move down the tree as shown in fig. 2 by hashed nodes. The procedure for finding radius using best first search is given in Algorithm. 4. The radius returned by this algorithm will be used in Algorithm. 1 .
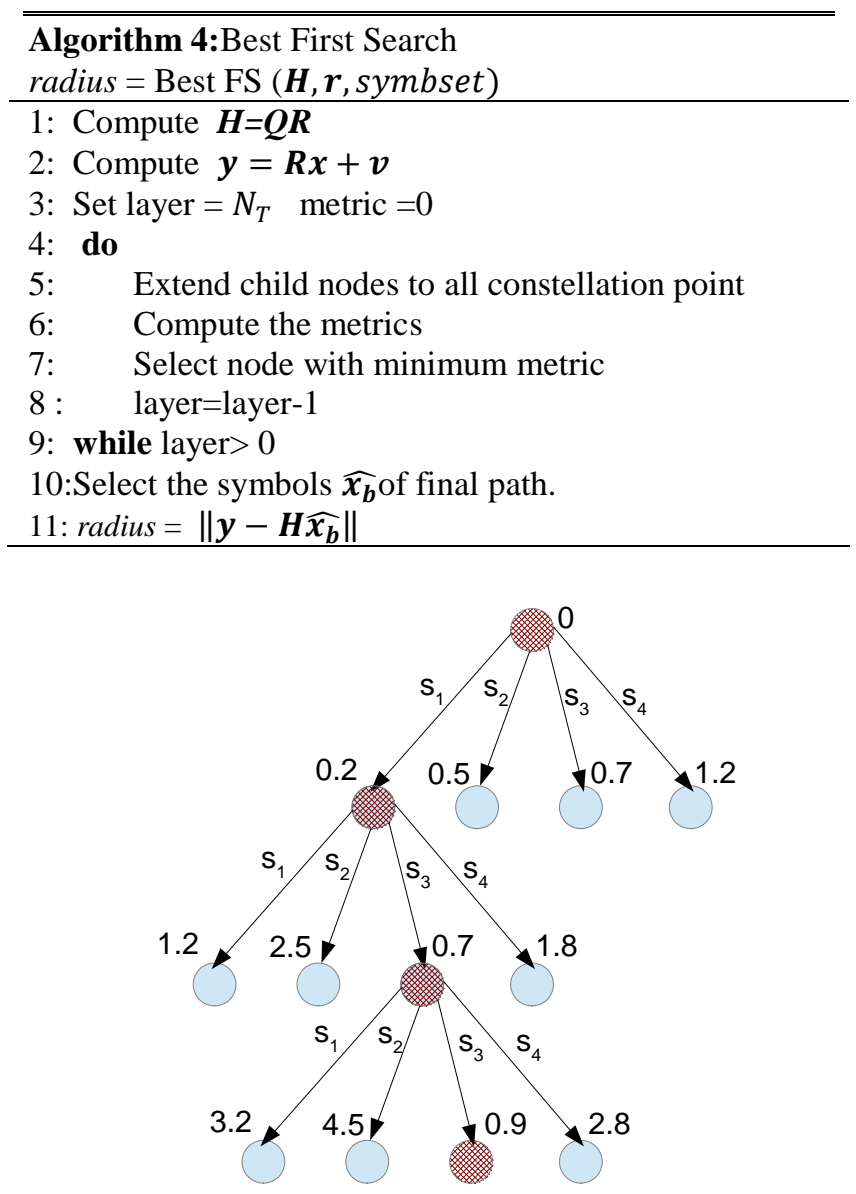

Fig. 2.Best First Search

\section{B. Modified QRD-M algorithm}

The computational complexity of QRD-M algorithm can be decreased by modifying the Algorithm 2 and 3. Here before extending the child nodes to all constellation points, the current metric of the node is compared with the maximum metric of the node in the next layer which is already computed.

For example, consider a 4X4 MIMO system with 4 constellation points (4-QAM modulation) and let $M=4$. At layer $=3$, there will be 4 surviving nodes as shown in fig. $3 \mathrm{a}$. We start with the first node which will have minimum metric. We extend all the child nodes to all 4 constellation points and calculate their metrics which will be metrics at layer=2 as shown in fig. 3b. These again will be arranged in sorted order. Now the next node at layer $=3$ will be extended if the metric of this node is less than the maximum of the metric of the nodes at layer $=2$. This procedure is continued for all the nodes in the current layer. Algorithm.5 gives the steps. 


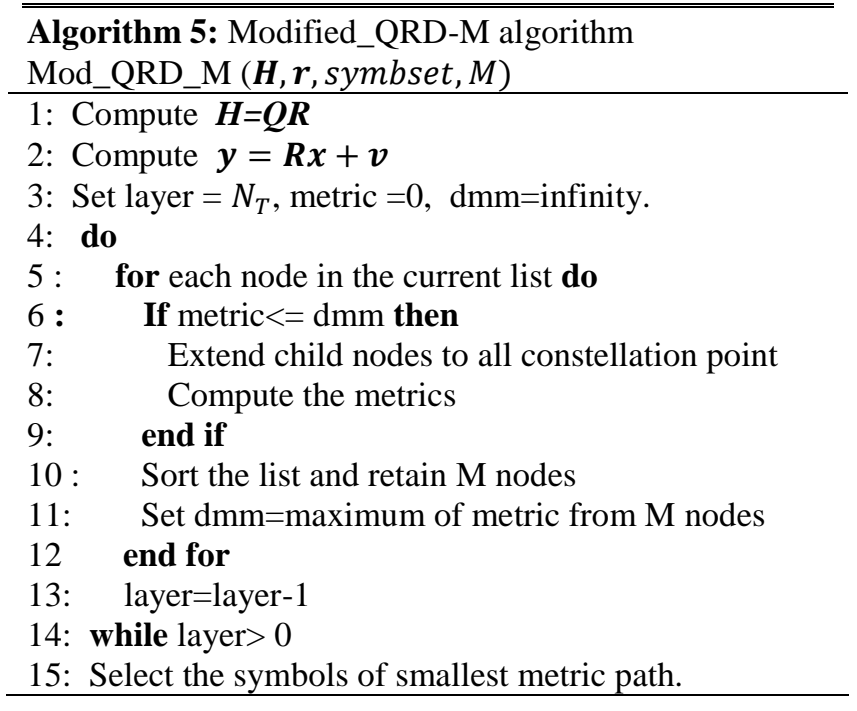

Above algorithm can be further speeded up by setting dmm value (step 11) equal to metric of some intermediate node instead of the maximum metric node. A similar modification can be done in Algorithm. 2.

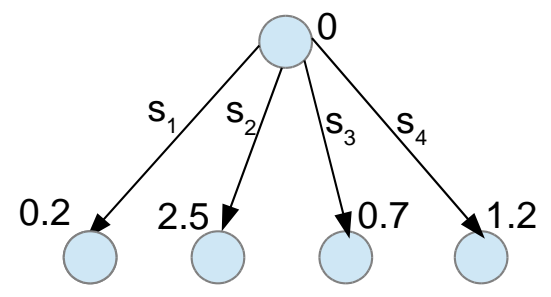

Fig. 3a.Modified QRD-M Algorithm Survival nodes at layer=3

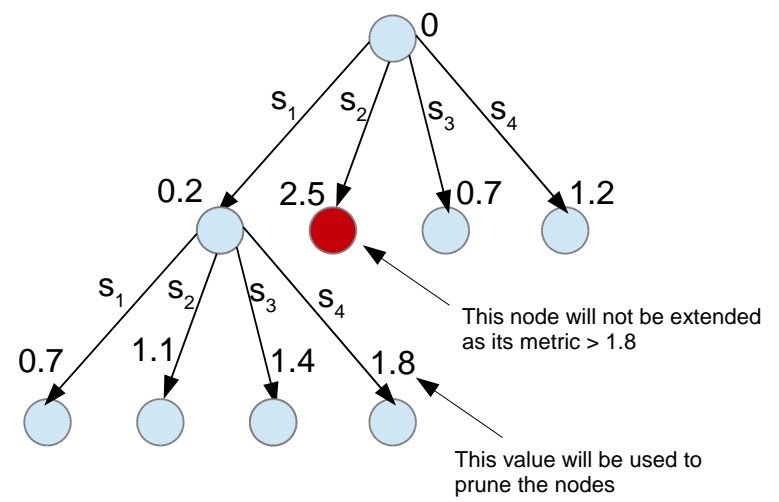

Fig. 3b. Modified QRD-M Algorithm, The minimum metric node $(0.2)$ in layer $=3$ will be extended. The maximum metric at layer $=2$ (1.8) will be used to decide whether the next node in the layer $=3$ is to be extended or not.

\section{RESULTS AND DISCUSSIONS}

The proposed algorithms were tested using simulation programs in Matlab. The average BER performance of MIMO systems was calculated using proposed algorithms. The computational complexity of the algorithms is also calculated using average number of nodes visited. The BER performance and computational complexity of proposed algorithms is compared with their original counterparts. Each simulated value in the results is obtained by averaging over 50 data packets of 100 frames each. The channel is assumed to be Rayleigh fading with additive white Gaussian noise.

\section{A. Performance of Sphere Decoding with best first Search}

Sphere decoding algorithms with infinite initial radius (SD), with Babai estimate (SD Babai Radius) and with best first search (SD Best FS Radius) techniques are shown in fig.4 for 4X4 MIMO SM system employing 4-QAM modulation. Fig. 5 shows the computational complexity of the algorithms in terms average number of nodes visited. It is seen that the BER performance of all the three algorithms is similar, whereas computational complexity of proposed technique is better in low SNR region. As discussed in section II, Babai estimate of the initial radius for low SNR will be affected by noise enhancement due to ZF detection. Hence the number of lattice points inside the hyper sphere will be more. The best first search technique tries to minimize the initial estimate of the radius and it is not affected by noise enhancement. In the high SNR regime, the noise is very small and the performance of $\mathrm{ZF}$ detector is improved giving rise to more accurate estimate of the initial radius. The best first search technique at high SNR has slight disadvantage over Babai estimate. Note that while calculating, the total average number of nodes visited, the nodes visited for calculating radius through best first search technique are also taken into account.

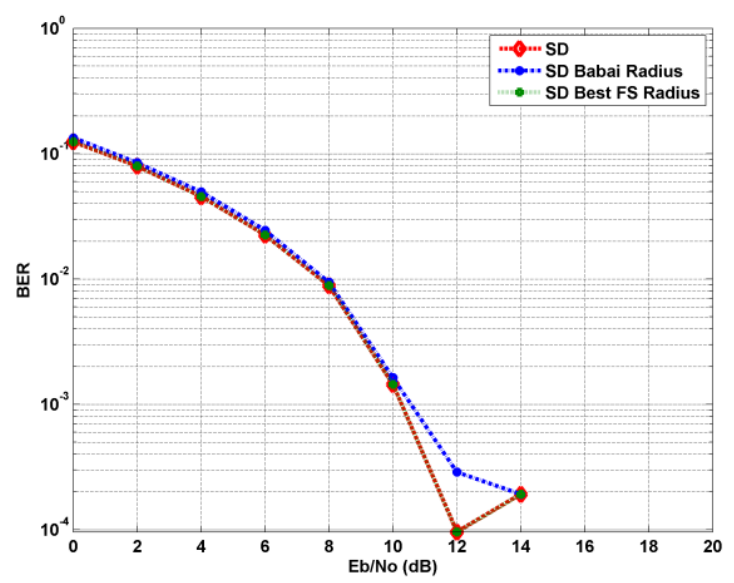

Fig. 4. Average BER Performance of sphere decoding algorithms, using different initial radius for 4X4 MIMO system with 4-QAM modulation.

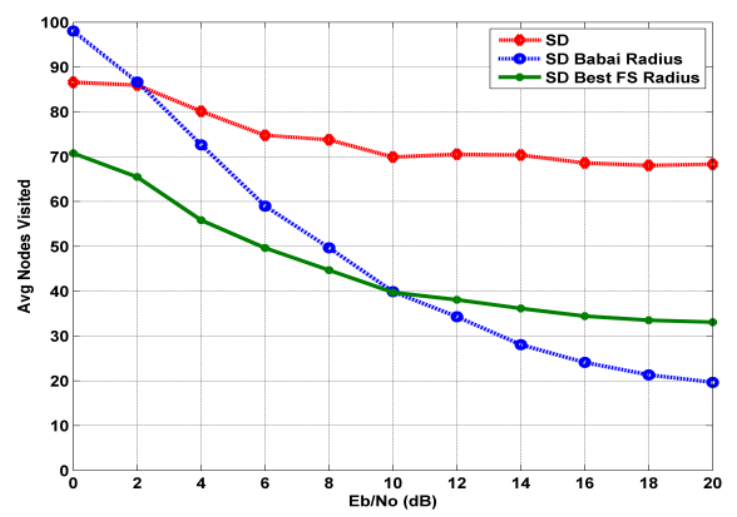

Fig. 5. Average Nodes Visited for sphere decoding algorithms using different initial radius for 4X4 MIMO with 4-QAM modulation 
Similar results can be obtained for 8X8 MIMO SM system with 4-QAM modulation. For large MIMO system, the proposed scheme is found to be superior to the existing algorithms in terms of performance and computational complexity. Fig. $6 \& 7$ show, BER performance and Average no of nodes visited plot of 4X4 MIMO SM system with 16QAM modulation. The results in the figures indicate that for higher modulation order, the proposed scheme is superior to the existing algorithms in terms of performance and computational complexity.

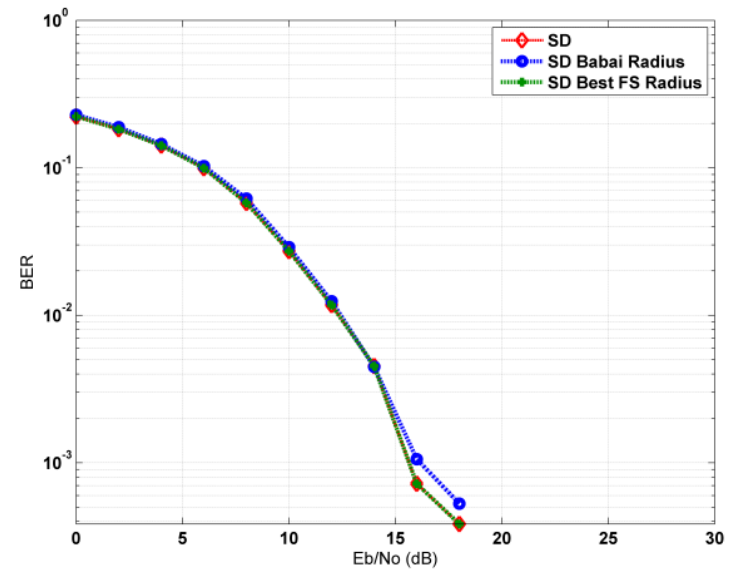

Fig. 6 Average BER Performance of sphere decoding algorithms, using different initial radius for 4X4 MIMO system with 16-QAM modulation

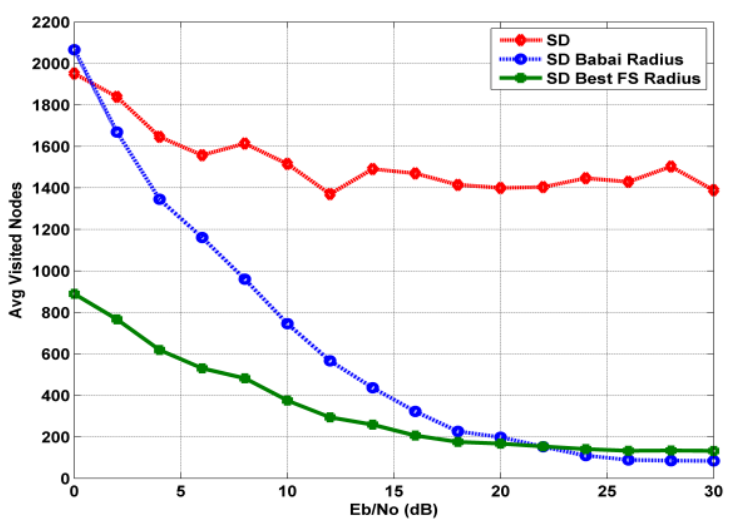

Fig. 7 Average Nodes Visited for sphere decoding algorithms using different initial radius for 4X4 MIMO system with 16QAM modulation.

\section{B. Performance of Modified QRD-M Algorithm}

Performance and complexity of modified QRD-M algorithm is studied for $4 \mathrm{X} 4$ and $8 \mathrm{X} 8 \mathrm{MIMO}$ systems. Fig. 8 shows, BER performance of 4X4 MIMO SM system. The BER is plotted for QRD-M algorithm with $\mathrm{M}=4$ and modified QRD-M with same value of M. The plot of SD with infinite initial radius is also given for comparison purpose. Fig. 9 shows the Computational complexity of the schemes in terms of average number of nodes visited.

It can be seen from the results of fig. 8 and 9 that the modified QRD-M algorithm has a slight edge in terms complexity over QRD-M algorithm whereas the BER performance remains same. Fig. 10 shows BER performance of the algorithms for 8X8 MIMO system with 4-QAM modulation and $\mathrm{M}=8$. In fig. 11 computational complexity in terms of average number of nodes visited is plotted for QRD$\mathrm{M}$ and modified QRD-M algorithms. The results obtained reiterate the fact that modified QRD-M algorithm has a slight edge over QRD-M method in terms of computational complexity.

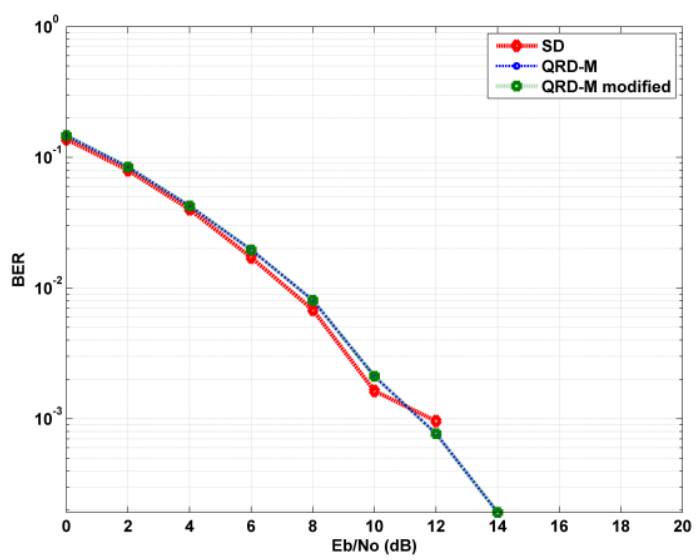

Fig. 8. Average BER Performance comparison of QRD-M and modified QRD-M algorithms 4X4 MIMO with 4-QAM modulation and $\mathrm{M}=4$.

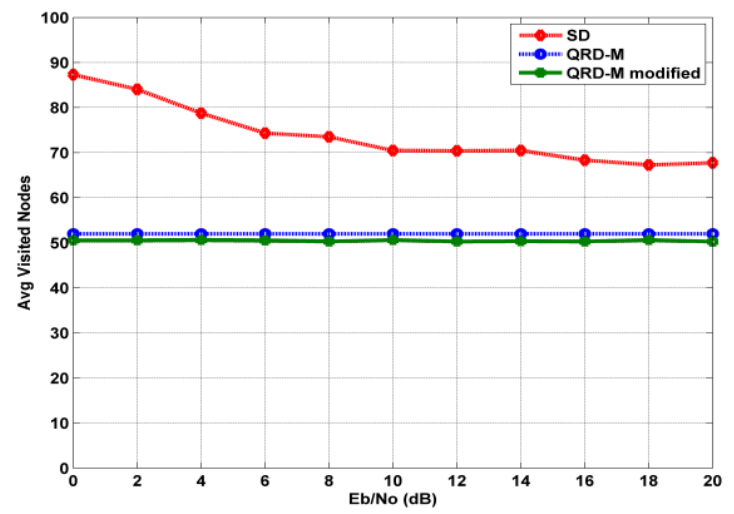

Fig. 9. Average Nodes Visited QRD-M and modified QRD-M algorithms 4X4 MIMO with 4-QAM modulation, and M=4.

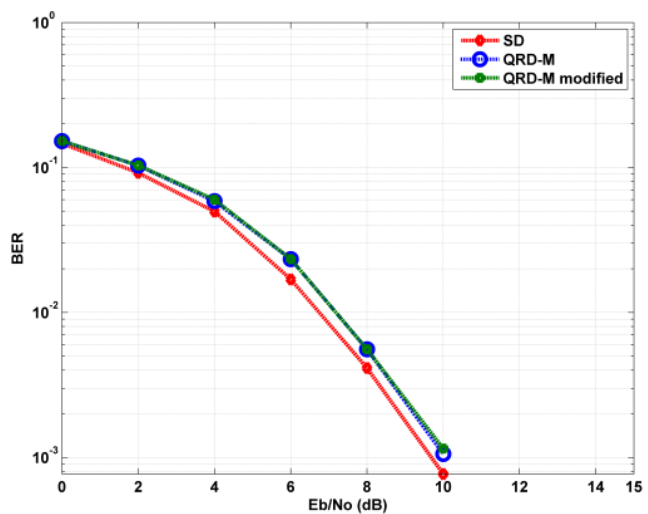

Fig. 10. Average BER Performance comparison of QRD-M and modified QRD-M algorithms 8X8 MIMO with 4-QAM modulation, and $\mathrm{M}=8$. 


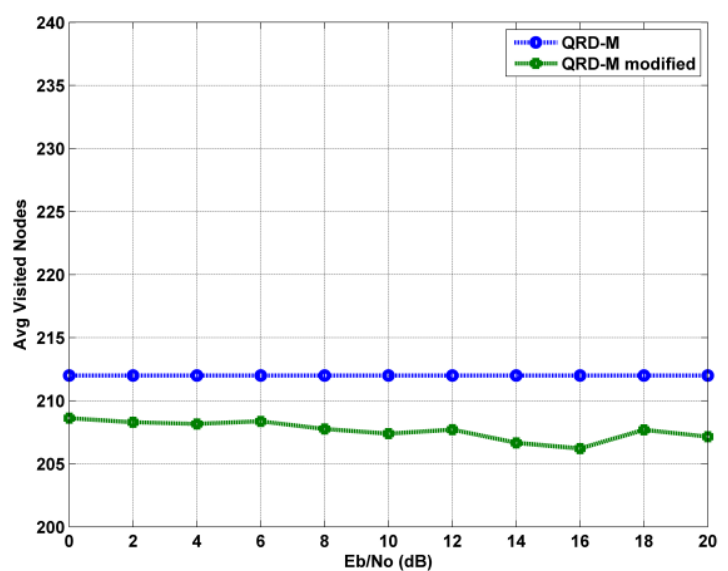

Fig. 11. Average Nodes Visited for QRD-M and modified QRD-M algorithms 8X8 MIMO with 4-QAM modulation and $\mathrm{M}=8$

\section{CONCLUSIONS}

Two reduced complexity tree search algorithms for detection of symbols in MIMO systems are proposed. The first algorithm is sphere decoding with initial estimate of radius calculated from best first search. The proposed algorithm demonstrates near ML performance with much lower computational complexity. In the second proposal, the QRD$\mathrm{M}$ algorithm is modified to prune the nodes in current layer based on metric calculated in the next layer. The BER performance of the proposed technique is same as QRD-M with reduced computational complexity. Future work on testing of these algorithms for soft sphere decoding can be carried out.

\section{REFERENCES}

[1] Jan Mietzner, Robert Schober, Lutz Lampe, Wolfgang H. Gerstacker, Peter A. Hoeher, "Multiple-Antenna Techniques for Wireless Communications - A Comprehensive Literature Survey," IEEE communications survey and tutorials,vol 2, no 2.pp. 87-105, Second quarter 2009

[2] Jeffrey G. Andrews, Arunabha Ghosh, and Rais Mohamed, "Fundamentals of WiMAX: Understanding Broadband Wireless Networking," Prentice Hall, 2007.

[3] Z. Xu and R. D. Murch, "Performance analysis of maximum likelihood detection in a MIMO antenna system," IEEE Trans. Comm., vol. 50, no. 2, pp. 187-191, Feb 2002.

[4] U. Fincke and M. Pohst, "Improved methods for calculating vectors of shortlength in lattice, including a complexity analysis", AMS Mathematics ofComputation, vol. 44, no. 170, pp. 463-471, April, 1985.

[5] C. P. Schnorr and M. Euchnerr. "Lattice basis reduction: improved practical algorithms and solving subset sum problems", Mathematical Programming, vol.66, pp.181-191, 1994.

[6] M. O. Damen, A. Chkeif, and J. C. Belfiore, "Lattice code decoder for space time codes," IEEE Communications Letters, vol. 4, pp. 161-163, May 2000.

[7] B. Hassibi and H. Vikalo, "On the Sphere Decoding Algorithm: Part I, The Expected Complexity", IEEE Trans. Sig. Process., vol. 53, no. 8 , pp. 2806-2818, August 2005 .

[8] Gajanan R Patil, Vishwanath K Kokate, "Simplified Implementation of Sphere Decoding Algorithms for MIMO Wireless Communication System”, In Proceedings of ICECCS, 19-21 Oct 2013, pp 128-137.
[9] J. Yue, K. J. Kim, J. D. Gibson, and R. A. Iltis, "Channel Estimation and DataDetection for MIMO-OFDM Systems," In Proc. GLOBECOM 2003, pp. 581-585, 2003.

[10] Gajanan R Patil, Vishwanath K Kokate, "Simplified Implementation of QRD-M Algorithms for MIMO Wireless Communication System,"CiiT Journal of Wireless Communication, vol. 5, No. 9, pp 392-397,Sept. 2013.

[11] Chin, W. H., "QRD based tree search data detection for MIMO communication systems," In Proceedings of IEEE 61st Vehicular Technology Conference, VTC 2005-Spring. 2005, vol. 3, pp. 1624 $1627,2005$.

[12] Li Liu, Jinkua Wang, Dongmei Yan, Fulai Liu, Xin Song, "Sorted QR Decomposition Based Tree Search Detection Algorithm for MIMO System," In Proceedings of Wireless 8th International Conference on Communications, Networking and Mobile Computing (WiCOM), pp. 1 $-4,2012$.

[13] Yu-Jin Song, Hyoung-Kyu Song, "Low complexity QRD-M algorithm based on LR-aided decoding for MIMO-OFDM systems," In Proceedings of IEEE 21st International Symposium on PIMRC, pp. 299 $-303,2010$

[14] Xinyu Mao, Yuxin Cheng, Lili Ma and Haige Xiang," Step reduced Kbest sphere decoding,", In Proceedings of IEEE Vehicular Technology Conference, VTC Fall 2012, pp. 1 - 4, 2012.

[15] Jin Sung, Kim, Sung-Hyun Moon, Inkyu Lee, "A new reduced complexity ML detection scheme for MIMO systems," IEEE Transactions on Communications, Volume: 58, pp.1302 - 1310, 2010.

[16] Bong-Seok Kim, Kwonhue Choi, "A Very Low Complexity QRD-M Algorithm Based on Limited Tree Search for MIMO Systems," In Proceedings of IEEE Vehicular Technology Conference, VTC Spring 2008, pp. 1246 - 1250, 2008.

[17] Li Wei, Chen Ming, Shixin Cheng, Haifeng Wang, “An Improved QRDM Algorithm in MIMO Communications," In Proceedings of IEEE Global Telecommunications Conference, GLOBECOM '07, pp. 4380 4384, 2007.

[18] Wei Peng, Shaodan Ma, Tung Sang Ng, Jiang Zhou Wang, “Adaptive QRD-M detection with variable number of surviving paths for MIMO systems," In Proceedings of International Symposium on Communications and Information Technologies, ISCIT '07, pp. 403 408, 2007.

[19] Sumei Sun, Yongmei Dai, Zhongding Lei, Higuchi Kenichi, "Pseudo Inverse MMSE Based QRD-M Algorithm for MIMO OFDM," In Proceedings of IEEE 63rd Vehicular Technology Conference, VTC 2006 Spring Volume: 3, pp.1545 - 1549, 2006.

[20] Yongmei Dai, Sumei Sun, Zhongding Lei, "A Comparative Study of QRD-M Detection and Sphere Decoding for MIMO-OFDM Systems," In Proceedings of IEEE 16th International Symposium on Personal, Indoor and Mobile Radio Communications, PIMRC 2005, Volume: 1, pp.186-190, 2005.

[21] L. Barbero, and J. Thompson, "Extending a fixed-complexity sphere decoder to obtain likelihood information for turbo-MIMO systems," In Proceedings of IEEE Transactions on Vehicular Technology vol. 57, no. 7, pp. 2804-2814., Sept. 2008.

[22] L. Barbero, and J. Thompson, "Fixing the complexity of the sphere decoder for MIMO detection," IEEE Transactions on Wireless Communications, vol. 7, no. 6, pp. 2131-2142, June 2008

[23] Qingwei Li, Corvallis, OR, Zhongfeng Wang, "Improved k-best sphere decoding algorithms for MIMO systems," In Proceedings of IEEE ISCAS 2006, May 2006.

[24] Zhan Guo and Peter Nilsson, "Algorithm and implementation of the Kbest sphere decoding forMIMO detection," IEEE Transactions onSelected Areas of Comm., vol. 24, No. 3, pp. 491-503,2006.

[25] Sandra Roger, Alberto Gonzalez,Vicenc Almenar, Antonio M. Vidal, "Practical aspects of pre-processing techniques for K-Best tree search MIMO detectors,"Computers and electrical engineering, vol.37, pp. 451460, 2011.

[26] Shen, Eltawil, Mondal, Salama, "A best-first tree-searching approach for ML decoding in MIMO system", IEEE ISCAS 2010, pp. 3333-3336. 


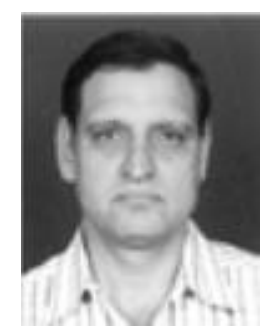

Gajanan R Patil was born in, India, in 1967. He received the B Tech in electronics engineering in 1989 from REC Calicut, ME in Electronics from Govt. College of Engg Pune in 1992. He is Pursuing his Ph.D. degree at Sinhgad College of Engg., Pune. Since 1997 he has been with Department of Electronics \& Telecommunication Engg at Army Institute of Technology, Pune where he is currently working as Associate Professor. $\mathrm{He}$ has published eight text books in the field of Electronics and Communication Engineering. His research interests are Wireless Communications and MIMO Channel Estimation \& detection, Coding.He is member of IEEE and IEEE Communication Society.

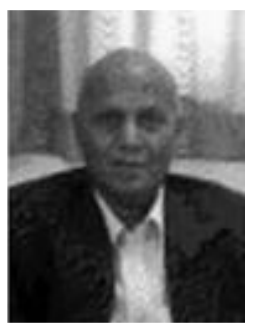

Prof. V. K. Kokate was born in India in 1950. He received his BE \& ME degree in Electronics \& Telecommunication Engg. from Govt. College of Engg., Pune in 1972 and 1977 respectively. $\mathrm{He}$ is currently $\mathrm{PhD}$ Guide at Sinhgad College of Engg Pune. He has more than 35 years of teaching experience. He was Director, Government College of Engg Pune from 2005 to 2007 . He has guided more than 10 $\mathrm{PhD}$ students. He has worked on number of committees of Government of Maharashtra and AICTE. He has published number of research papers in National, International Conferences and Journals. His research interests include Microwave, antenna and wireless communications. 